Computing (MIT Media Laboratory), pp. 132-139, Los Alamitos, CA: IEEE Computer Society Press, Aug. 1998.

[31] N. S. Shenck and J. A. Paradiso, "Energy scavenging with shoe-mounted piezoelectrics," IEEE Micro, vol. 21, no. 3, pp. 30-42, May 2001.

[32] P. Glynne-Jones, S. P. Beeby, and N. M. White, "Towards a piezoelectric vibration-powered microgenerator," IEEE Sci. Meas. Technol., vol. 148, no. 2, pp. 68-72, Mar. 2001.

[33] S. Roundy, "The power of good vibrations," Lab Notes-Research From the College of Engineering, vol. 2, no. 1, Berkeley, CA: Berkeley Press, Jan. 2002.

[34] T. G. Engel, "Energy conversion and high power pulse production using miniature piezoelectric compressors," IEEE Trans. Plasma Sci., vol. 28, no. 5, pp. $1338-1340$, Oct. 2000

[35] N. W. Haggod IV et al., "Development of micro-hydraulic transducer technology," presented at the 10th Int. Conf. on Adaptive Structures and Technologies, Paris, France, Oct. 1999.

[36] C. S. McDowell, "Implanted Bone Stimulator and Prosthesis System and Method of Enhancing Bone Growth," U.S. Pat. 6143 035, Nov. 7, 2000.

[37] C. C. Enger and J. H. Kennedy, "Piezoelectric power sources utilizing the mechanical energy of the human heart," presented at the 16th Annual Conf. Engineering in Medicine and Biology, Baltimore, MD, Nov. 1963.

[38] — " "An improved bioelectric generator," Trans. Am. Soc. Artif. Intern. Organs, vol. 10, pp. 373-377, Oct. 1964

[39] W. H. Ko, "Piezoelectric energy converter for electronic implants," presented at the 19th Annual Conf. Engineering in Medicine and Biology, San Francisco, CA, Nov. 1966.

[40] G. H. Meyers et al., "Biologically energized cardiac pacemakers," IEEE Trans. Bio-Med. Eng., vol. BME-10, p. 83, Apr. 1963.

[41] G. H. Meyers et al., "Biologically energized cardiac pacemakers," Amer. J. Med. Electron., vol. 3, pp. 233-236, Oct/Dec. 1964.

[42] ISO 14243-1:2002(E) Implants for surgery-wear of total knee-joint prostheses-Part 1: Loading and displacement parameters for wear-testing machines with load control and corresponding environmental conditions for test. Int. Org. for Standardization, 03, 2002.

[43] V. E. Bottom, Introduction to Quartz Crystal Unit Design. New York: Van Nostrand Reinhold, 1982.

[44] W. G. Cady, Piezoelectricity: An Introduction to the Theory and Applications of Electromechanical Pheonomena in Crystals. New York: Dover, 1964, vol. $1 \& 2$

[45] M. Rossi, Acoustics and Electroacoustics. Norwood, MA: Artech House, 1988

[46] "Effects of high static stress on the piezoelectric properties of transducer materials," vol. 33, no. 10, pp. 1339-1344, Oct. 1961.

[47] M. D. Hill, G. S. White, and C. Hwang, "Cyclic damage in lead zirconate titanate," J. Amer. Ceram. Soc., vol. 79, no. 7, pp. 1915-1920, Jul. 1996.

[48] M. G. Cain, M. Stewart, and M. G. Gee, "Degradation of Piezoelectric Materials," National Physical Laboratory Management Ltd., Teddington, Middlesex, U.K., TW11 0LW, NPL Rep. SMMT (A) 148, 1999.

[49] G. Yang, S. F. Liu, W. Ren, and B. K. Mukherjee, "Uniaxial stress dependence of the piezoelectric properties of lead zirconate titanate ceramics," "in Active Materials: Behavior and Mechanics," SPIE Proceedings Bellingham, WA, vol. 3992, pp. 103-113, 2000.

[50] "Domain processes in lead zirconate and barium titanate ceramics," vol. 30, no. 11, pp. 1804-1810, Nov. 1959.

[51] F. Lowrie, M. Cain, and M. Stewart, "Time dependent behaviour of piezoelectric materials," National Physical Lab. Management Ltd., Teddington, U.K., TW11 OLW, NPL Rep. SMMT (A) 151, 1999.

[52] D. M. Breiner "Deterioration of soft PZT due to cyclic loading," M.S. thesis, Univ. of Nebraska-Lincoln, 1997.

\section{Denoising Jet Engine Gas Path Measurements Using Nonlinear Filters}

\author{
Rajeev Verma and Ranjan Ganguli
}

\begin{abstract}
Traditionally, linear filters have been used to smooth time series of gas path measurements before performing fault detection and isolation. However, linear filters can smooth out sharp trend shifts in the signal and are also not good at removing outliers. Since most fault detection and isolation algorithms are optimized for Gaussian noise, they can show performance degradation when outliers are present. In this study, numerical results with simulated data for engine deterioration and abrupt fault show that the nonlinear rational filter with median preprocessor are useful for gas turbine health monitoring applications resulting in noise reduction of $73 \%-96 \%$ while preserving signal features and removing outliers.
\end{abstract}

Index Terms-Fault diagnosis, gas turbines, signal processing.

\section{INTRODUCTION}

Health monitoring applications typically involve detection and isolation of a system fault based on a comparison between a "good" baseline system and a "damaged" system [1]. A health signal can be interpreted as a measurement delta between the damaged measurement $z^{(d)}$ and undamaged measurement $z^{(u)}$ and written as $\Delta=z^{(d)}-z^{(u)}$. Under ideal conditions, when a system has no fault, $\Delta=0$. When a fault occurs, $\Delta$ assumes a nonzero value whose magnitude depends of the size and location on the fault. In this idealized system, the nonzero value of the measurement deviation, along with other measurement deviations, can be used to detect and isolate the fault.

Studies of gas turbine data have shown two main features of the health signal $\Delta: 1$ ) most major problems in the engine are caused by a "single fault" which is preceded by a sharp trend shift [2] and 2) long-term deterioration in the engine causes a low-order polynomial variation in the measurements with time, with a linear polynomial being a very good approximation [3]. However, noise and outliers are present in the gas turbine health signals. Therefore, processing of health signals is often done before using fault isolation methods using linear filters [4]. However, linear filters smooth out the sharp edges in the signal that contain important information about the fault initiation time as well as repair events and could be used for fault isolation applications. Furthermore, linear filters are not good at removing outliers in the data. For commercial aircraft engines, only few data points are received for each flight. Therefore, it is important to keep the forward data point requirement to a minimum. In this paper, we explore nonlinear rational and median filters with a low-time delay for gas turbine applications.

\section{GAS TURBInE DiAgnOSTICS}

Fig. 1 shows a schematic of a turbofan engine which has five modules: fan, low-pressure compressor (LPC), high-pressure compressor (HPC), low-pressure turbine (LPT), and high-pressure turbine (HPT). Faults in the gas turbine engine cause efficiency deterioration for the engine modules. The engine state is monitored using at least the four basic sensors: exhaust gas temperature (EGT), fuel flow (WF), low rotor speed (N1), and high rotor speed (N2). The measurement deltas $\Delta \mathrm{EGT}, \Delta \mathrm{WF}, \Delta \mathrm{N} 1$, and $\Delta \mathrm{N} 2$ are used for estimating the engine

Manuscript received March 18, 2003; revised July 9, 2003. Recommended by Technical Editor J. van Amerongen.

R. Verma is with the Advanced Engineering, Ashok Leyland Ltd., Chennai 600035, India (e-mail: rajeevverma.alc@ashokleyland.com).

R. Ganguli is with the Department of Aerospace Engineering, Indian Institute of Science, Bangalore 560012, India (e-mail: ganguli@ aero.iisc.ernet.in).

Digital Object Identifier 10.1109/TMECH.2005.852454 


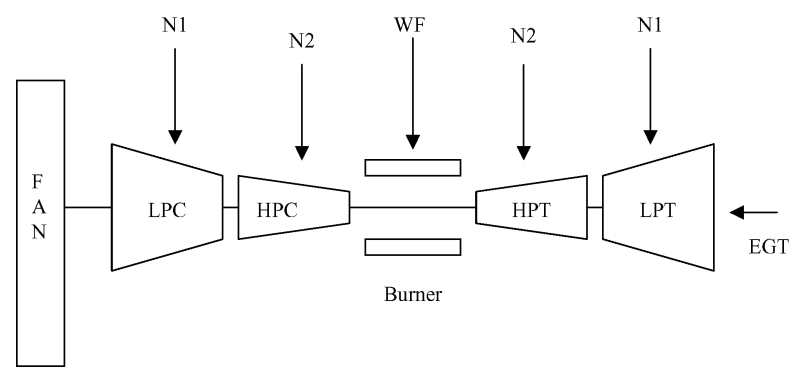

Fig. 1. Schematic view of turbofan gas turbine engine modules and sensors.

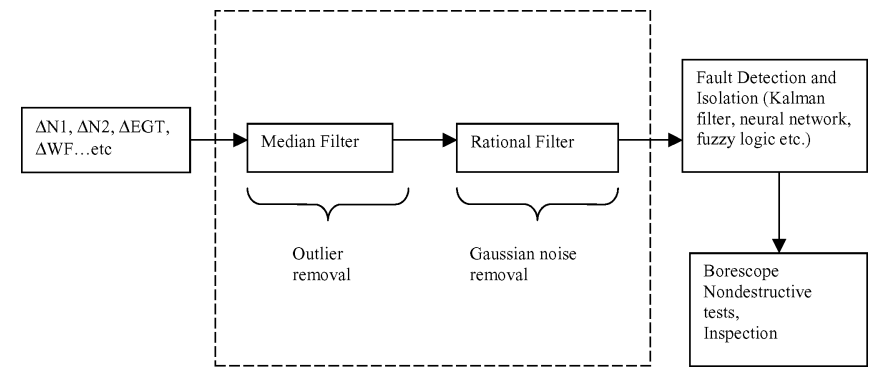

Fig. 2. Schematics of noise and outlier removal in gas turbine diagnostics process.

state. Fig. 2 shows a schematic of the gas turbine diagnostic process. It is clear that if the fault module is correctly identified, the cost of maintenance of the airline comes down.

\section{TEST SIgnAlS}

The gas path signals can be modeled using 1) step and 2) ramp edges. Consider the time series for 25 points shown in the ideal step signal in Fig. 3. This step signal simulates an abrupt fault. The onset of the fault(s) is at discrete time $k=12$. The noisy signal can be expressed as

$$
\Delta=\Delta^{0}+\alpha \varepsilon+\theta
$$

where $\Delta^{0}$ is the pure signal, $\varepsilon$ and $\theta$ are added Gaussian noise and outliers, respectively, and $\alpha$ is a parameter that allows control of the level of noise in the noisy health signal $\Delta$. Fig. 3 shows the ideal step signal along with a noisy test signal with $\alpha=0.2$. The outlier signal contains five points represented by $\theta=-1$ at $k=7, \theta=0.75$ at $k=10, \theta=-0.75$ at $k=14, \theta=1$ at $k=18$, and $\theta=-1.5$ at $k=22$. These outliers are placed in an arbitrary way along the time series and do not follow any noise model. Lu [5] calls these "wild points" which tend to occur in gas path sensor measurements. Fig. 4 shows the ideal and noisy test signal for a ramp edge simulating engine deterioration. The outliers are placed at the same location as for the step signal.

Table I shows the fingerprint chart for a large commercial engine which are the fault signatures of the engine at a given steady flight condition and relate the faults in a given module to changes in the gas path measurements [4]. For the fingerprints shown in Table I, the measurement uncertainties for $\Delta \mathrm{EGT}, \Delta \mathrm{WF}, \Delta \mathrm{N} 2$, and $\Delta \mathrm{N} 1$ are $4.23 \mathrm{C}, 0.50 \%, 0.17 \%$, and $0.25 \%$, respectively [4]. These numbers were obtained by a study of airline data. Using these numbers for the four measurements, the signal to noise ratios are obtained by dividing the fingerprints in Table I with the corresponding measurement uncertainty. These results are shown in Table II where it can be seen that the signal-to-noise ratios (SNRs) range from a low of 0.56 to a high of 7.84. Since the ideal test signals in Figs. 3 and 4 have a maximum value
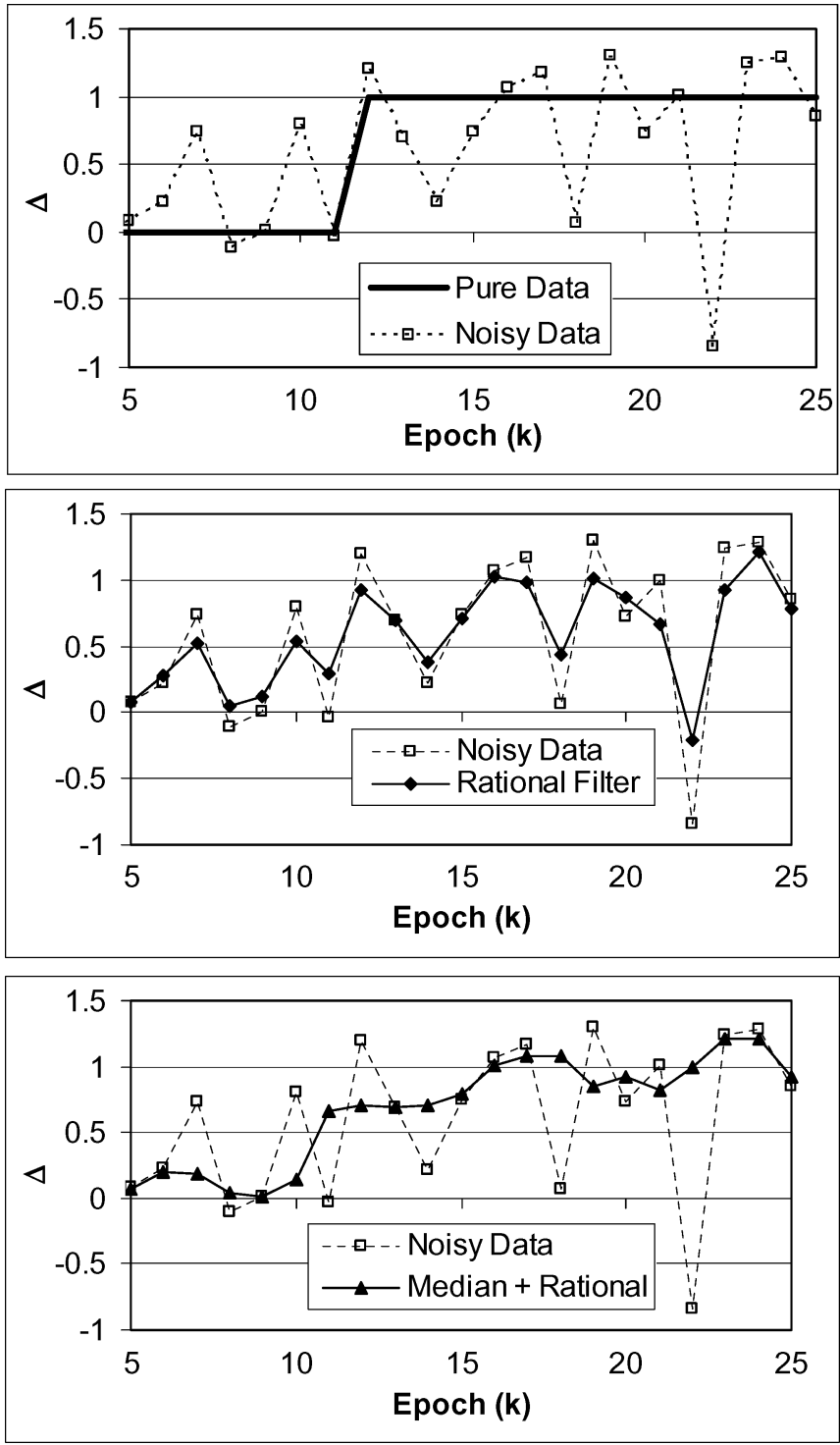

Fig. 3. Ideal, noisy and filtered signal for engine "abrupt fault."

TABLE I

FINGERPRINTS FOR SELECTED GAS TURBINE FAULTS $\eta=-2 \%$

of one, a noise level of 0.1 leads to an SNR of 10 and a noise level of 0.4 leads to an SNR of 2.5. Also note that the ideal signals vary from zero in the initial stages to values between 0 and 1 for the ramp edge in Fig. 4. Therefore, a wide range of signal to noise ratio are addressed using the variation in $\alpha$ from 0.1 to 0.4 . 

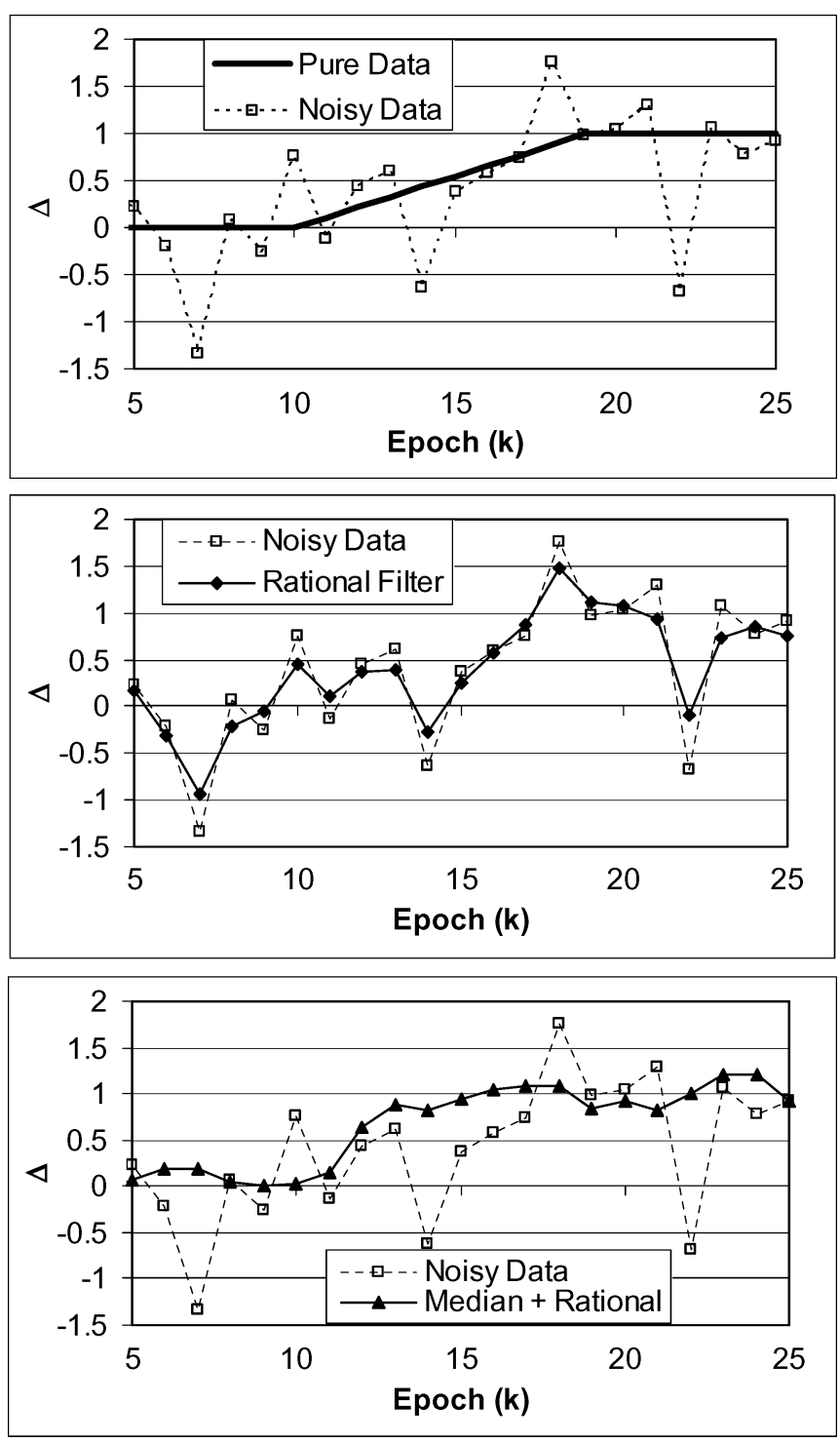

Fig. 4. Ideal, noisy and filtered signal for engine "deterioration."

TABLE II

SNRs FOR FINGERPRINTS OF GAS TURBINE FAULTS IN TABLE I

\begin{tabular}{ccccc} 
Faults & $\Delta E G T$ & $\Delta W F$ & $\Delta N 2$ & $\Delta N 1$ \\
\hline High Pressure Compressor & 3.215 & 3.2 & 0.65 & 0.40 \\
High Pressure Turbine & 5.15 & 5.16 & 6.65 & 0.60 \\
Low Pressure Compressor & 2.14 & 2.64 & 3.35 & 1.12 \\
Low Pressure Turbine & 0.56 & 3.84 & 7.47 & 7.84 \\
Fan & 1.82 & 2.80 & 3.47 & 5.40 \\
\hline
\end{tabular}

The mean square error (MSE) provides information about the filter accuracy and is defined as

$$
\operatorname{MSE}=\sum_{i=1}^{N} \frac{\left(\Delta-\Delta^{0}\right)^{2}}{N}
$$

where $N$ is the number of samples, $\Delta$ is the noisy or filtered signal value and $\Delta^{0}$ is the ideal or pure signal value. The noise reduction is defined as

$$
\Theta^{(\mathrm{MSE})}=100 \frac{\mathrm{MSE}^{(\text {noisy })}-\mathrm{MSE}^{(\text {filtered })}}{\mathrm{MSE}^{(\text {noisy })}} .
$$

\section{BACKGROUND ON FILTERING}

The filters used in this study are the rational filter and the median filter.

\section{A. Rational Filter}

The rational filter is defined [6] by a rational function, which is the ratio of two polynomials.

$$
\hat{\Delta}_{k}=\frac{\Delta_{k-1}+\Delta_{k+1}+\Delta_{k}\left[\kappa\left(\Delta_{k-1}-\Delta_{k+1}\right)^{2}+\frac{1}{w}-2\right]}{\kappa\left(\Delta_{k-1}-\Delta_{k+1}\right)^{2}+\frac{1}{w}} .
$$

Here $\hat{\Delta}_{k}$ is the filtered signal value at discrete time $k$. The values of data at $k-1, k$, and $(k+1)$ th time are $\Delta_{k-1}, \Delta_{k}$, and $\Delta_{k+1}$. The parameters $\kappa$ and $w$ takes positive values and are used to control the filter. The rational filter differs from the linear finite impulse response (FIR) filter mainly for the scaling which is introduced on the $\Delta_{k-1}$ and $\Delta_{k+1}$ terms. Such terms are divided by a factor proportional to the edge sensing term. When $\kappa=0$, the rational filter acts as the following linear filter:

$$
\hat{\Delta}_{k}=w\left(\Delta_{k-1}+\Delta_{k+1}\right)+(1-2 w) \Delta_{k} .
$$

The sum of the coefficients or weights of the above filter is one. The filter shows low pass behavior for $0<w<1 / 3$. For $w=1 / 3$, the filter becomes a moving average filter. When $\kappa \rightarrow \infty$, the filter has no effect, and $\hat{\Delta}_{k} \cong \Delta_{k}$. For intermediate values of $\kappa$, the $\left(\Delta_{k-1}-\Delta_{k+1}\right)^{2}$ term perceives the presence of a detail and accordingly reduces the smoothing effect of the operator.

\section{B. Median Filter}

The three point median filter can be written as [4]

$$
\hat{\Delta}_{k}=\operatorname{median}\left(\Delta_{k-1}, \Delta_{k}, \Delta_{k+1}\right) .
$$

The median is a selection filter which means that its output is limited to one of the input samples. Therefore, the median is not very good at removing Gaussian noise since each element of the input sample contains Gaussian noise. However, the median filter is conceptually very simple though long-length median filters involve sorting operations that can be computationally expensive. Therefore, in this paper, we use only three point median filter to minimize forward point requirements and keep the computational expenses down.

\section{Median Plus Rational Filter}

The hybrid filter we propose preprocesses the signal with a median filter before using the rational filter and is shown in schematic form in Fig. 2 inside the dotted lines. First, the measurement delta is passed through the median filter.

$$
\begin{aligned}
y_{k-1} & =\operatorname{median}\left(\Delta_{k-2}, \Delta_{k-1}, \Delta_{k}\right) \\
y_{k} & =\operatorname{median}\left(\Delta_{k-1}, \Delta_{k}, \Delta_{k+1}\right) \\
y_{k+1} & =\operatorname{median}\left(\Delta_{k}, \Delta_{k+1}, \Delta_{k+2}\right) .
\end{aligned}
$$

During this phase, the outliers in the data are removed. In the next phase, the median preprocessed data is sent through the rational filter. 
TABLE III

Noise Reduction With Filters For Engine Abrupt Fault Signal BaSed ON MSE

\begin{tabular}{|c|c|c|c|c|c|c|c|}
\hline$\alpha$ & 0.10 & 0.15 & 0.20 & 0.25 & 0.30 & 0.35 & 0.40 \\
\hline Rational & 46.93 & 46.95 & 46.99 & 47.05 & 47.13 & 47.21 & 47.29 \\
\hline Median & 86.39 & 83.21 & 79.41 & 75.36 & 71.43 & 67.94 & 65.04 \\
\hline Median+Rational & 88.37 & 85.96 & 83.11 & 80.11 & 77.23 & 74.68 & 72.58 \\
\hline
\end{tabular}

The rational filter can be defined using the outputs of the median filter from (7) as

$$
\begin{aligned}
d_{k} & =\kappa\left(y_{k-1}-y_{k+1}\right)^{2}+\frac{1}{w} \\
\hat{\Delta}_{k} & =\frac{y_{k-1}+y_{k+1}+y_{k}\left[d_{k}-2\right]}{d_{k}} .
\end{aligned}
$$

The "median plus rational" approach is computationally efficient and has a two point time delay. In this study, $\kappa$ is fixed at 0.01 and $w$ at 0.16 which are optimal for a signal contaminated with Gaussian noise [6]. This is a good assumption for the "median plus rational" approach as the data is first subjected to a median filter which removes outliers and the rational filter is then used on a signal with trend shift and Gaussian noise.

\section{NUMERICAL RESULTS}

Numerical results are obtained using the test signal shown in Figs. 3 and 4, which contain the step signal and the ramp signal simulating abrupt fault and engine deterioration, respectively. The noisy data shown in these figures uses $\alpha=0.2$ and added outliers which were discussed earlier. Figs. 3 and 4 also show the results of processing the noisy signal using the rational and median plus rational filters, respectively.

From Fig. 3, we see that the rational filter is able to preserve the trend shift while reducing Gaussian noise to some extent, but is unable to discard the outliers. The "median plus rational" approach results in the outliers being removed from the signal and Gaussian noise being reduced while preserving the trend shift. Fig. 4 shows that for a linear signal, the rational filter is unable to remove outliers. However, the "median plus rational" approach results in outlier removal along with some removal of Gaussian noise. The visual quality of the signals is considerably improved after the application of the "median plus rational" filter. Since monitoring "trend plots" of gas path measurement deltas form an important diagnostic tool for airline powerplant engineers, the use of the nonlinear filters for smoothing the data can greatly increase their capability of finding faults by visual inspections of the gas path sensor data itself.

The above results are qualitative and provide visual information about the filters. However, they represent only one of many possible noisy data samples. To obtain quantitative results, 1000 samples of noisy data are created about the ideal step and ramp signals in Figs. 3 and 4 and the noise reduction after filtering is calculated. Tables III and IV show the noise reduction based on the mse for the step signal and the ramp signal, respectively.

The noise level added to the ideal signal varies from low $(\alpha=0.10)$ to high $(\alpha=0.40)$. The rational filter reduces noise by $47 \%$ for the step signal and by about $48 \%$ for the ramp signal. The noise reduction is almost constant across the noise levels. The median filter reduces noise level by $65-86 \%$ for the step signal and 7096 for the ramp signal. For the median filter, the noise reduction decreases with increasing levels of Gaussian noise in the data. The median filter works better when
TABLE IV

NOISE REduction With Filters FOR ENGine Deterioration Signal BASED ON MSE

\begin{tabular}{|c|c|c|c|c|c|c|c|}
\hline$\alpha$ & 0.10 & 0.15 & 0.20 & 0.25 & 0.30 & 0.35 & 0.40 \\
\hline Rational & 48.44 & 48.39 & 48.34 & 48.3 & 48.27 & 48.25 & 48.23 \\
\hline Median & 95.38 & 91.56 & 86.91 & 82 & 77.31 & 73.13 & 69.60 \\
\hline Median+Rational & 96.18 & 93.38 & 89.96 & 86.33 & 82.85 & 79.74 & 77.11 \\
\hline
\end{tabular}

the data has outliers and low levels of Gaussian noise. The "median plus rational" filter reduces noise by $7388 \%$ for the step signal and $77-$ $96 \%$ for the ramp signal. The "median plus rational" filter gives more noise reductions than the median filter at all noise levels. However, the advantage increases at higher levels of Gaussian noise where the random noise removing ability of the rational filter is useful.

\section{CONCLUDING REMARKS}

Simulated health monitoring test signals are used to evaluate the denoising capability of the "median plus rational" filter for smoothing health signals. The rational filter, when used alone, is not good for outlier removal though it preserves the edges in the health signal. If the data are preprocessed by a median filter and then sent through a rational filter, both outliers and Gaussian noise is removed while preserving the edges in the signal which are often precursors to abrupt faults. The "median plus rational" filter results in noise reduction of $73-96 \%$ for the noisy signals and is also conceptually simple and computationally efficient when implemented in a small window of three points as suggested in this paper. Furthermore, the filter has a two point time delay, making it suitable for jet engine diagnostics where few points are obtained for each flight and the cost of transmitting additional points is high. Using these filters with real data is a subject of future work.

\section{REFERENCES}

[1] R. Isermann, "Supervision, fault-isolation and fault diagnosis methodsAn introduction," Cont. Eng. Practice, vol. 5, no. 5, pp. 639-652, 1997.

[2] H. Depold and F. D. Gass, "The application of expert systems and neural networks to gas turbine prognostics and diagnostics," ASME J. Eng. Gas Turbines Power, vol. 121, no. 4, pp. 607-612, 1999.

[3] K. Mathioudakis, Ph. Kamboukos, and A. Stamasis, "Turbofan performance deterioration tracking using non-linear models and optimization techniques," ASME J. Turbomach., vol. 124, no. 4, pp. 580-587, 2002.

[4] R. Ganguli, "Data rectification and detection of trend shifts in jet engine gas path measurements using median filters and fuzzy logic," ASME J. Eng. Gas Turbines Power, vol. 124, no. 4, pp. 809-816, 2002.

[5] P. J. Lu, T. C. Hsu, M. C. Zhang, and J. Zhang, "An evaluation of engine fault diagnostics using artificial neural networks," ASME J. Eng. Gas Turbines Power, vol. 123, no. 2, pp. 340-346, 2001.

[6] G. Ramponi, "The rational filter for image smoothing," IEEE Signal Process. Lett., vol. 3, no. 3, pp. 63-65, 1996. 